\title{
New Starch Hybrids via Etherification of Poly (Acrylamide)-Starch Copolymers with Acrylamide
}

\author{
A. Hebeish, A. Mousa ${ }^{*}$, M.A. Ramadan ${ }^{\#}$ and A. Saleh \\ Textile Research Division, National Research Centre and \\ *Chemistry Department, Faculty of Science, Ain Shams \\ University, Cairo, Egypt.
}

\begin{abstract}
OVEL starch hybrids containing acrylamide (Aam) moieties in monomeric (i.e carbamoylethyl groups) and polymeric (poly acrylamide) forms were synthesized. Thus, starch was first polymerized with acrylamide to yield poly (Am)-starch composite and poly (Aam)starch graft copolymer which represent the total polymerization products before and after removal of the homopolymer, respectively. The composite and the copolymer were then carbamoyethylated via reaction with Aam. Beside the carbamoylethyl groups, carboxyethyl groups were inevitably formed during carbamoylethylation. This and the onset of such modification on the rhelogical properties of the so synthesized starch hybrids signify the following. a) The extents of carbamoylethylation of the composite and the copolymer were much lower than native starch; b) The magnitude of poly(Am) content in the form of graft or homopolymer adversely affects the carbamoylethylation reaction; c) before carbamoylethylation, the composite, the copolymer and native starch exhibited non-Newtonian thixotropic behavior, d); after carbamoylethylation the etherified products were characterized by pseudoplastic behavior. The apparent viscosity of starch, starch composite and starch copolymer decreased significantly after carbamoylethylation but with the certainty that the apparent viscosity increased by increasing the carbamoylethyl and carboxyethyl groups in these starch hybrids.
\end{abstract}

Keywords: Starch, Copolymer, Acrylamide, Etherification and Composite.

A great deal of research and technical work has been carried out with a view to improve the physical and chemical characteristics of starch as a prerequisite to promote utilization of the latter. The studies reported so far are, in essence, dealt with swelling ${ }^{(1)}$, oxidation ${ }^{(2-4)}$, hydrolysis, esterification ${ }^{(5)}$, etherification ${ }^{(6-9)}$, grafting $^{(10,11)}$ and cross-linking of starch ${ }^{(12)}$. Also reported are the combined hydrolysis (or oxidation) and grafting of starch ${ }^{(10,11)}$. Major properties affected by such modifications are solubility, viscosity performance and resistance to ageing of native starch and products derived from modification.

This work is undertaken with a view to synthesize new starch hybrids containing acrylamide (Aam) moieties in monomeric and polymeric forms. To achieve the goal, poly (Aam)-starch graft copolymer was first synthesized then

\footnotetext{
\#Corresponding auther E. mail: amaramadan1@ hotmail.com
} Tel: 00201227108920 Fax: 0020233363261 
subjected to carbamoylethylation. The latter was studied under different conditions in order to establish the most appropriate conditions for carbamoylethylation of the starch copolymer. Conditions so established were employed for carbamoylethylation of the starch composite. The work was extended to study the hydrolysis of the amide groups of poly (Aam) to carboxyl groups during the carbamoylethylation treatment and the onset of this on the rheological properties of the starch products.

\section{Materials}

\section{Experimental}

Maize starch was supplied by the Egyptian Starch and Glucose Manufacturing Company, Cairo, Egypt. Acrylamide (Aam), sodium hydroxide, potassium persulphate, sodium thiosulphate, hydrochloric acid, sodium carbonate, ethanol, and acetone are of laboratory grade chemicals.

\section{Grafting method}

Unless otherwise indicated, the grafted starch was prepared by mixing $10 \mathrm{~g}$ starch with $100 \mathrm{ml}$ water in a flask. The latter was placed in a thermostatic water bath adjusted at $65^{\circ} \mathrm{C}$, and the starch /water mixture therein was subjected to continuous mechanical stirring $(200 \mathrm{rpm})$ to get homogenized slurry. Aam monomer [50\% based on weight of starch (ows)] was then added to the homogenized slurry while stirring. After that, the components of redox initiator system were added simultaneously. This system consisted of potassium persulphate and sodium thiosulphate with concentration of $1.1 \mathrm{mmol} / \mathrm{l}$ and $3.3 \mathrm{mmol} / \mathrm{l}$, respectively. Polymerization system was allowed to proceed for $60 \mathrm{~min}$ at $65^{\circ} \mathrm{C}$ with continuous stirring. The material to liquor ratio (M: L.R) was 1:10. After 60 min-reaction, polymerization was ceased by adding $10 \mathrm{ml}$ of $0.1 \%$ hydroquinone solution. At this end, the polymerization products in the flask were neutralized using $1 \%$ sodium carbonate solution then poured over ethanol, where a precipitate was formed ${ }^{(12)}$. The so obtained precipitate is referred to as "composite". The latter consists of poly (Aam)-starch graft copolymer, poly (Aam) (homopolymer), oxidized starch, and unreacted starch. The homopolymer was removed from the composite according to reported method ${ }^{(13)}$.

\section{Carbamoylethylation method}

Unless otherwise indicated, the experimental technique adopted for carbamoylethylation of starch, starch composite and starch copolymer was as follows: Sodium hydroxide solution (5\% ows), was added gradually to $100 \mathrm{~g}$ starch in a flask with continuous mechanical stirring $(200 \mathrm{rpm})$. This was followed by addition of acrylamide (100\% ows) while stirring. The latter continued till the flask contents were completely homogenized. The flask was then transferred to a thermostatic water bath and the reaction was allowed to proceed under continuous mechanical stirring at $30^{\circ} \mathrm{C}$ for $90 \mathrm{~min}$. At this end, the reaction product was precipitated and washed several times in ethanol then dried at $50^{\circ} \mathrm{C}$ in an oven ${ }^{(14)}$. 


\section{Cooking process}

An aqueous dispersion of starch or the modified starches under investigation was prepared at a concentration of $10 \%$ solid content. The dispersion was then homogenized before heating by making use of a mechanical stirrer $(250 \mathrm{rpm})$ for $10 \mathrm{~min}$. Cooking was effected by heating the sample under continuous stirring in thermostatic water bath where the temperature was raised from $25^{\circ} \mathrm{C}$ to $95^{\circ} \mathrm{C}$ in a rate of $2.5^{\circ} \mathrm{C}$ per min then kept constant at $95^{\circ} \mathrm{C}$ for 30 min cooking to bring about a paste. Temperature of this paste was lowered to $25^{\circ} \mathrm{C}$; meanwhile stirring was reduced to the minimum degree within $15 \mathrm{~min}$.

Analysis

The carboxyl content of starch and the modified starches before and after etherification was determined according to a reported method ${ }^{(15)}$. This method is based on acid-base titration.

The nitrogen content of starch and modified starch before and after etherification was determined according to Kjeldhal method ${ }^{(16)}$.

The reaction efficiency was calculated based on the sum of both carboxyl and nitrogen contents.

The rheological properties were measured using Roto- Visco RV 20 under the following experimental conditions. Rate of shear was between 129-1290 S${ }^{-1}$ and temperature $80^{\circ} \mathrm{C}$. The apparent viscosity was calculated using the following formula $\eta=S \tau / D$ where:

$\eta=$ Apparent viscosity (mPa.)

$\mathrm{D}=$ Rate of shear $\quad\left(\mathrm{S}^{-1}\right)$

$\mathrm{S} \tau=$ Shear stress $\quad(\mathrm{mPa} . \mathrm{S})$

\section{Results and Discussion}

\section{Tentative mechanisms}

In a system containing starch, acrylamide, sodium hydroxide and water, the following reactions are expected to occur:

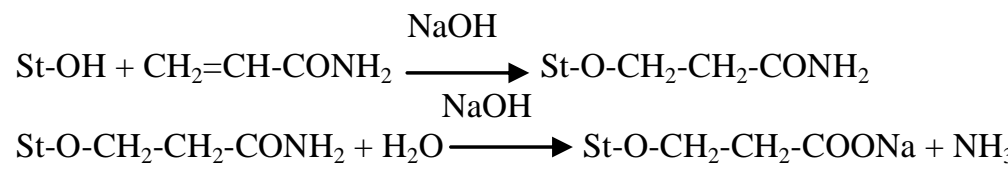

$$
\mathrm{CH}_{2}=\mathrm{CH}-\mathrm{CONH}_{2}+\mathrm{H}_{2} \mathrm{O} \stackrel{\mathrm{NaOH}}{\longrightarrow} \mathrm{CH}_{2}=\mathrm{CH}-\mathrm{COONa}+\mathrm{NH}_{3}
$$

Similar reactions would take place when starch (St-OH) is replaced by poly (Aam)-starch graft copolymer. 
Furthermore, water adds to acrylamide in the presence of alkali to give amidohydroxyethylene which still further reacts with acrylamide yielding bis-2 carbamoylethyl ether ${ }^{(1)}$.
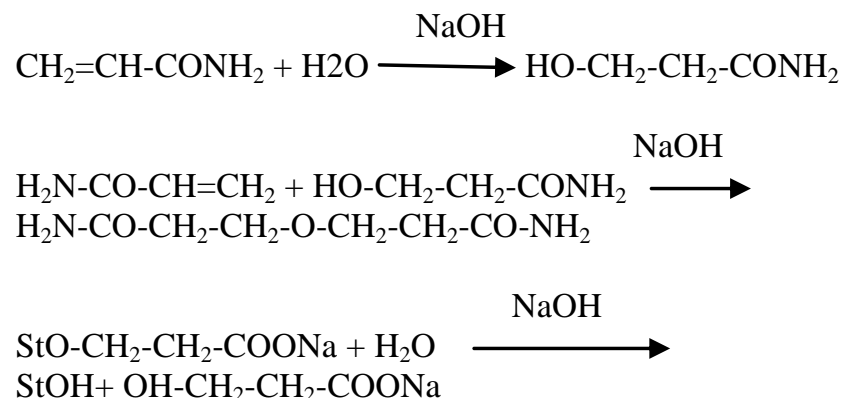

Poly (Aam)-starch graft copolymer was reacted with acrylamide under a variety of conditions. This was done to discover the most appropriate conditions for carbamoylethylation of the copolymer which would also be applied to the poly (Aam)-starch composite.

\section{Sodium hydroxide concentration}

Figure 1A shows the effect of sodium hydroxide concentration on the extent of carbamoylethylation reaction, expressed as nitrogen percent, when poly (Aam)-starch graft copolymer with original nitrogen content of $8.3 \% \mathrm{~N}$ was carbamoylethylated. The results disclose that the extent of the carbamoylethylation reaction increases significantly by increasing sodium hydroxide concentration up to $5 \%$ on weight of starch (ows) then decreases thereafter. Sodium hydroxide seems to perform: i) catalyzing the carbamoylethylation reaction, ii) enhancing the swellability of the copolymer thereby improving the extent of the reaction through better diffusion of reactants and iii) hydrolyzing the amide group in acrylamide, poly (Aam)-starch graft copolymer and the carbamoylethylated products derived thereof. It is logical that factors (i) and (ii) would act in favor of carbamoylethylation whereas factor (iii) would exert a reverse action.

As shown in Fig. 1A the efficiency of the carbamoylethylation reaction exhibits a trend which is similar to that obtained with the extent of the reaction. Maximum reaction efficiency is achieved at $\mathrm{NaOH}$ concentration of $5 \%$ ows. Above this concentration lower reaction efficiency is achieved by a virture of the progressive action of hydrolysis at higher $\mathrm{NaOH}$ concentrations which may be accompanied by de-etherification. It is understandable that the reaction efficiency is based on the total amount of both nitrogen and carboxyl content of the copolymer after carbamoylethylation.

The pronounced effect of hydrolysis of the amide groups of the copolymer under the action of increasing sodium hydroxide concentration is obvious when the results of carboxyl percent are considered. The carboxyl percent $(\mathrm{COOH} \%)$

Egypt. J. Chem. 56, No. 4 (2013) 
in Fig 1A increases significantly by increasing sodium hydroxide concentration up to $10 \%$ ows then continues to increase but marginally by further increasing $\mathrm{NaOH}$ concentration to $20 \%$. It is also noted that the lower values of $\mathrm{N} \%$ (measure for extent of reaction) are encountered by higher values of carboxyl \%( measure for the extent of hydrolysis) within the $\mathrm{NaOH}$ concentration range of 5 $20 \%$ (ows).

It is as well to emphasize that presence of measurable amount of carboxyl groups in the copolymer after carbamoylethylation regardless of $\mathrm{NaOH}$ concentration used is indicative of concurrent carbamoylethylation and hydrolysis. At higher $\mathrm{NaOH}$ concentrations the decrement in extent of the carbamoylethylation reaction is outweighed by increment in the extent of hydrolysis reaction.

\section{Acrylamide concentration}

Figure 1B shows the effect of acrylamide concentration on the extent and efficiency of the carbamoylethylation of poly (Aam)-Starch graft copolymer.

It is seen that the extent and efficiency of the carbamoylethylation reaction increase substantially as the acrylamide concentration increases up to $100 \%$ ows. Further increase in the acrylamide concentration causes no increase in the extent and efficiency of the reaction as evidenced by the constant values of $\mathrm{N} \%$ and Carboxyl \%.

The enhancement in the extent and efficiency of the reaction by increasing acrylamide concentration up to certain limit could be associated with greater availability of acrylamide molecules in the vicinity of the copolymer at higher acrylamide concentration. Since the residual starch hydroxyls in the copolymer are immobile, their reaction with acrylamide and sodium hydroxide would essentially depend upon availability of both reactant molecules in the proximity of hydroxyl of poly (Aam)-Starch graft copolymer molecule. On the other hand, leveling off of the carbamoylethylation after a certain acrylamide concentration could be interpreted in terms of reduction of reactive sites, i.e. hydroxyl groups, on the copolymer molecules. This means that there is a certain amount of accessible hydroxyl groups in the molecular structure of poly (Aam)-starch graft copolymer. Once these groups are blocked by the carbamoylethylation groups, further reaction becomes difficult under condition used.

Figure 1B shows the dependence of the carboxyl \% (measure of the extent of conversion of amide groups via alkaline hydrolysis) of the copolymer on the acrylamide concentration. Evidently, the carboxyl \% increases by increasing acrylamide concentration up to $100 \%$ ows then remains almost constant upon further increase in the acrylamide concentration; similar to the extent and efficiency of the reaction. It follows from this that the magnitude of carbamoylethylation of the copolymer determines the magnitude of the alkaline 
hydrolysis. Stated in other words, alkaline hydrolysis occurs concurrent with carbamoylethylation and is favored under conditions which favor carbamoylethylation.
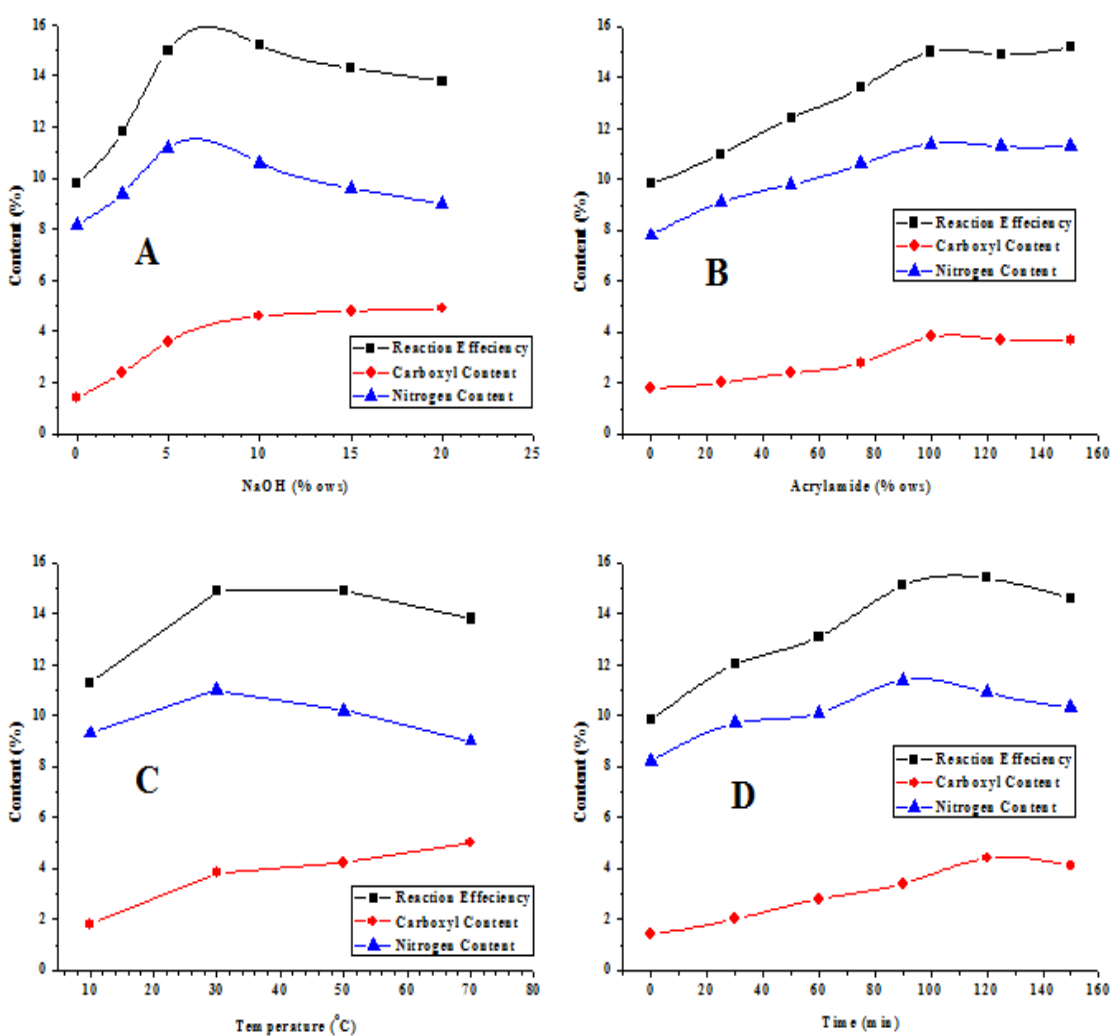

Fig. 1. Different factors affecting the carbamoylethylation of poly(Aam)-starch graft copolymer

A. Effect of sodium hydroxide concentration (Aam: $100 \%$ ows; Temp.: 30 ; Time: 90 min.; M: L.R.: 1:10)

B. Effect of acrylamide concentration (NaOH: 5 \%ows; Temp.: 30 ${ }^{\circ} \mathrm{C}$; Time: 90 min.; M: L.R.: 1:10)

C. Effect of temperature (NaOH: 5 \%ows; Aam: 100 \%ows; Time: 90 min.; M: L.R.: 1:10)

D. Effect of time (NaOH: 5 \%ows; Aam: 100 \%ows; Temp.: 30C; M: L.R.: 1:10)

\section{Effect of temperature}

Figure 1C shows the effect of temperature on the extent of the carbamoylethylation reaction, expressed as $\mathrm{N} \%$, and efficiency of the reaction, based on nitrogen and carboxyl contents of poly (Aam)-starch graft copolymer. The results signify that the extent and efficiency of carbamoylethylation attain temperature their maximum at $30^{\circ} \mathrm{C}$; then start to decrease with increasing the temperature above $30^{\circ} \mathrm{C}$. It is logical that higher temperature would enhance the extent and rate of alkaline hydrolysis which, as already stated, takes place alongside the carbamoylethylation. Alkaline hydrolysis converts the amide to carboxyl groups. Indeed the results of carboxyl \% shown in Fig. 1C and the trend obtained when these results are plotted versus the temperature are in full

Egypt. J. Chem. 56, No. 4 (2013) 
agreement with the statement emphasizing conversion of the amide to carboxyl groups. However, the contribution of the de-etherification in reaction efficiency cannot be ruled out.

\section{Effect of duration}

Figure 1D shows the effect of reaction time on the extent and efficiency of carbamoylethylation of poly (Aam)-starch graft copolymer. It is seen that when plotted versus time, the extent and efficiency of carbamoylethylation follow similar trend, the extent of carbamoylethylation and efficiency of etherification increase by prolonging the duration of the etherification to reach the highest values after $90 \mathrm{~min}$. Thereafter, they decrease indicating that longer than $90 \mathrm{~min}$ reaction time is meaningless since it adversely affects the carbamoylethylation reaction. Although the reaction time provides better opportunity for appropriate contact among reactants for the reaction to proceed yet longer time seems to promote alkaline hydrolysis not only of the newly introduced amide in the form of carbamoylethyl groups but also the amide groups of the graft in the poly (Aam)starch graft copolymer. In combination with this is the plot shown in Fig. 1D of the carboxyl \% versus the reaction time. As is evident, the carboxyl \% of the etherified copolymer increases by increasing the duration of carbamoylethylation upto 120 min. Further prolonging the duration tends to decrease the carboxyl \% suggesting de-etherification. That is, splitting off of the carboxyethyl groups of the carbamoylethylated poly (Aam)-starch graft copolymer seems to occur under the influence of longer time in presence of alkali. As a result, the carboxyl \% tends to decrease.

\section{Optimum conditions}

Based on the result of Fig. 1 (A, B, C and D) discussed above, it may be concluded that the most appropriate conditions for carbamoylethylation of poly (Aam)-starch graft copolymer are as follows: sodium hydroxide 5\% ows, acrylamide $100 \%$ ows, reaction time $90 \mathrm{~min}$., and temperature $30^{\circ} \mathrm{C}$.

\section{Nature of substrate}

In this study the optimum conditions achieved above for carbamoylethylation of the copolymer were applied to different substrates, namely, unmodified starch as well as starch copolymers and starch composites having different nitrogen percent and carboxyl contents as indicated in Table 1 . The study was undertaken with a view to shed insight on the effect of structural differences among these substrates on the extent of carbamoylethylation. Also studied was the carboxyethylation process occurring concurrently with carbamoylethylation through conversion of the carbamoylethyl groups as well as the amide groups of the copolymer and composite to carboxyethyl groups and the onset of this on the rheological properties of the carbamoylethylated products.

\section{Nitrogen content}

Figure 2 shows the nitrogen percent before and after carbamoylethylation of starch composites and starch graft copolymers. The composites and copolymers 
were prepared using different concentrations of acrylamide. Obviously, the copolymers are more susceptible to carbamoylethylation than the composites. This is observed regardless of the magnitude of poly (Aam) of both substrates. Presence of poly (Aam) in the form of homopolymer which is most probably in intimate association with other components of the composites seems to be responsible for this. The homopolymer may act as a diffusion barrier for and /or mask starch hydroxyls thereby preventing their ability and accessibility to undergo the carbamoylethylation reaction.

TABLE 1. Nitrogen percent of starch- poly Aam composite and copolymer before and after etherification with Aam in alkaline medium.

\begin{tabular}{|c|c|c|c|c|}
\hline \multirow{3}{*}{$\begin{array}{c}\text { [Aam] } \\
\text { \%ows }\end{array}$} & \multicolumn{4}{|c|}{ Nitrogen content (\%) } \\
\hline & \multicolumn{2}{|c|}{ Poly (Aam)-starch composite } & \multicolumn{2}{|c|}{ Poly (Aam)-starch graft copolymer } \\
\hline & BC & $\mathbf{A C}$ & BC & $\mathbf{A C}$ \\
\hline 5 & 2.3 & 5.3 & 1.3 & 4.5 \\
\hline 10 & 3.9 & 6.7 & 2.6 & 5.6 \\
\hline 20 & 6.8 & 9.3 & 5.3 & 7.9 \\
\hline 30 & 8.3 & 10.3 & 6.8 & 9.0 \\
\hline
\end{tabular}

Polymerization conditions: $\left[\mathrm{K}_{2} \mathrm{~S}_{2} \mathrm{O}_{8} / \mathrm{Na}_{2} \mathrm{~S}_{2} \mathrm{O}_{3}\right]=1.1 / 3.3 \mathrm{mmol} / \mathrm{l}$; time $=60 \mathrm{~min}$; temp $=60^{\circ} \mathrm{C}$; M:L.R $=1: 10$ Etherification conditions: $[\mathrm{NaOH}]=5 \%$ ows; $[$ Aam $]=100 \%$ ows; time $=90 \mathrm{~min}$; temp $=30^{\circ} \mathrm{C} ; \mathrm{M}: \mathrm{L} . \mathrm{R}=1: 10$. $\mathrm{BC}$ : before carbamoyletylation $\mathrm{AC}:$ After carbamoyletylation

\section{Carboxyl content}

Figure 3 shows the effect on the carboxyl content of the magnitude of poly (Aam) content in the copolymer and the composite substrates. Different poly (Aam) contents were obtained using different acrylamide concentrations in the polymerization medium during preparation of both substrates. The carboxyl contents of the copolymer and composite were determined before and after the copolymer and the composite were reacted with acrylamide in alkaline medium. It is seen that: a) the carboxyl content is directly related to the acrylamide concentration used in the polymerization which, in turn, determines the poly (Aam) content, b) the composite exhibits higher carboxyl content than does the copolymer and c) carboxyl content is much higher after than before etherification, i.e. reacting the copolymer and the composite with acrylamide in alkaline medium. These findings clearly indicate that introduction of acrylamide monomeric (as carbamoylethyl groups) or polymeric (as polyacrylamide) in the molecular structure of starch is associated with carboxyethyl groups. The latter are formed via hydrolysis of the amide groups in the acrylamide moietycontaining products; the larger the amount of acrylamide moieties, the greater the carboxyl content. 
In combination with this is the alkaline condition of carbamoyethylation which would favour carboxyl group formation through alkaline hydrolysis of the amide groups. This is, rather, obvious in Fig. 3 where enhanced in the carboxyl content of the composite and the copolymer is observed after carbamoylethylation.

\section{Reaction efficiency (total extent of etherification)}

The total extent of the etherification reaction occurring between acrylamide and starch, starch composite or starch copolymer can be expressed as the sum of nitrogen and carboxyl group which are taken as a measure for carbamoylethylation and carboxyethylation extents, respectively. Results of the sum of both nitrogen and carboxyl group (expressed as mmol/100g sample) of the etherified starch products are set out in Table 2 . The results bring into focus two main conclusions.

TABLE 2. Total extent of etherification (reaction efficiency) obtained when the starch, starch graft copolymer and starch composite were independently reacted with Aam in alkaline medium.

\begin{tabular}{|c|c|c|c|c|c|c|c|c|c|c|c|c|}
\hline \multirow{3}{*}{ 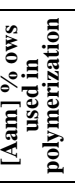 } & \multicolumn{6}{|c|}{ Poly (Aam)-starch composite } & \multicolumn{6}{|c|}{ Poly (Aam)-starch graft composite } \\
\hline & & & $\mathrm{BC}$ & & & $\mathbf{A C}$ & & & BC & & & $\mathrm{AC}$ \\
\hline & $\mathbf{z}$ & $\begin{array}{l}\pi \\
8 \\
8\end{array}$ & 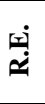 & $\mathbf{z}$ & 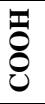 & 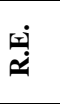 & $\mathbf{z}$ & $\begin{array}{l}\text { : } \\
8\end{array}$ & 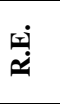 & $\mathbf{z}$ & $\begin{array}{l}\text { ¿̊ } \\
8\end{array}$ & 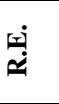 \\
\hline n & $\underset{\sigma}{t}$ & 官 & g & శે & 8 & ॄे & ๙ & $\stackrel{\text { iे }}{\text { ते }}$ & ป & $\vec{ల}$ & in & $\stackrel{m}{m}$ \\
\hline 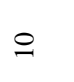 & ปे & $\stackrel{n}{m}$ & $\stackrel{n}{0}$ & જे & $\stackrel{n}{2}$ & 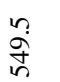 & 气े & $\dot{\vec{m}}$ & $\stackrel{\text { वें }}{2}$ & \& & రु & दुव \\
\hline กิ & \& & $\begin{array}{l}\tilde{N} \\
\text { di }\end{array}$ & $\begin{array}{l}\infty \\
i \\
i n\end{array}$ & t్ర & $\stackrel{2}{2}$ & & ڤે & $\frac{\dot{m}}{m}$ & $\begin{array}{l}\stackrel{t}{\circ} \\
\forall\end{array}$ & 总 & $\infty$ & $\overrightarrow{\mathrm{d}}$ \\
\hline 只 & $\mathscr{\infty}$ & $\begin{array}{l}\infty \\
\dot{m}\end{array}$ & & 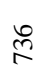 & $\stackrel{\text { }}{\sigma}$ & ఏ్ & 文 & ij & $\stackrel{\substack{\infty \\
n}}{n}$ & fo & बू. & 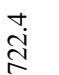 \\
\hline
\end{tabular}

Nitrogen content $(\mathrm{N})$ and carboxyl content $(\mathrm{COOH})$ expressed as mmol/100g. sample;

Reaction Efficiency (R.E.) $=$ sum of $\mathrm{N}+\mathrm{COOH}(\mathrm{mmol} / 100 \mathrm{~g}$ sample).

Polymerization conditions : $\left[\mathrm{K}_{2} \mathrm{~S}_{2} \mathrm{O}_{8} / \mathrm{Na}_{2} \mathrm{~S}_{2} \mathrm{O}_{3}\right]=1.1 / 3.3 \mathrm{mmol} / \mathrm{l}$; time $=60 \mathrm{~min}$; temp $=60^{\circ} \mathrm{C}$; M:L.R $=1: 10$. carbamoylethylation conditions: $[\mathrm{NaOH}]=5 \%$ ows; $[$ Aam $]=100 \%$ ows; time $=90 \mathrm{~min}$; temp $=30^{\circ} \mathrm{C}$; M:L.R =1:10.

$\mathrm{BC}$ : before carbamoyletylation $\mathrm{AC}$ : After carbamoyletylation

Firstly, the reaction efficiency of the etherification reaction decreases by the increase in acrylamide concentration indicating that side reactions previously indicated are more pronounced at higher acrylamide concentration than does the carbamoylethylation reaction. Secondly, the starch graft copolymer displays greater reaction efficiency than the starch composite most probably owing to the presence of the homopolymer, the latter is present in intimate association with the copolymer in the composite and may act as a diffusion barrier for the etherifying agents thereby decreasing the reaction efficiency of etherification. 


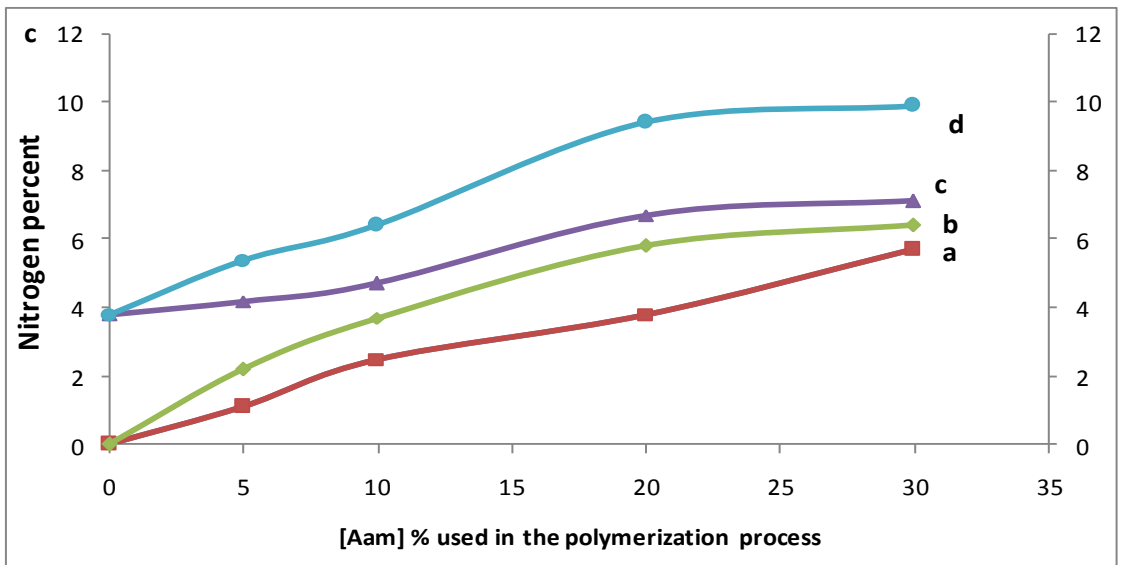

Fig. 2. Dependence of the nitrogen percent of starch- poly (Aam) composite and the copolymer before and after carbamoylethylation on acrylamide concentration used for preparation of the composite.

a-Poly (Aam)-starch graft copolymer b-Poly(Aam)-starch composite c-Carbomoylethyl poly(Aam) - starch graft copolymer

d-Carbomoylethyl poly(Aam) - starch composite

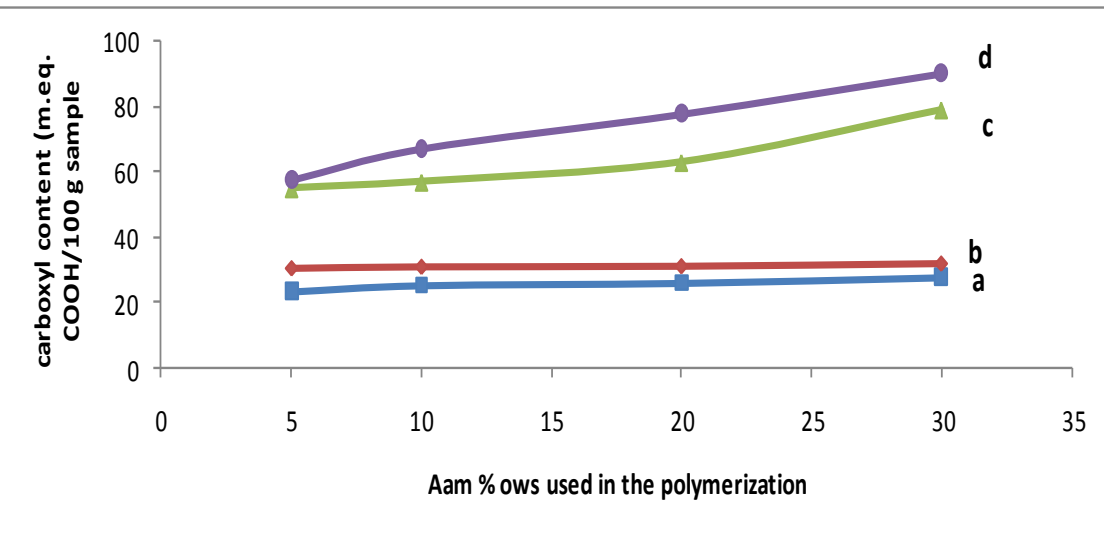

Fig. 3. Dependence of the carboxyl content of starch -poly (Aam) composite and copolymer before and after carbamoylethylation on acrylamide concentration used for synthesis of the composite. a-Poly(Aam)- starch graft copolymer b- Poly(Aam)- starch composite c-Carbomolethyl poly(Aam)- starch composite d-Carbomoylethyl-poly(Aam)- starch graft copolymer

Rheological properties

shear stress versus rate of shear

It is well established that the correlation between shear stress $(\mathrm{S} \tau)$ and rate of shear (D) defining the flow curve or flow behavior of a liquid is graphically displayed in diagram of $(\mathrm{S} \tau)$ on the ordinate and $\mathrm{D}$ on the abscissa. This diagram is called flow curve. In this work the flow curve diagrams were produced directly

Egypt. J. Chem. 56, No. 4 (2013) 
from HAAKE ROTO Visco RV 20 viscometer equipment at $80^{\circ} \mathrm{C}$, for native maize starch, poly (Aam)-starch graft copolymer, starch composite when Aam was used at concentrations of 10, 20 and $30 \%$ ows for preparation of the composite and copolymer. It was found from the flow curve diagrams (not shown to save space) that the shear stress increases as the rate of shear increases from zero to $1290 \mathrm{~s}^{-1}$. Opposite situation was encountered by decreasing the rate of shear from the maximum $1290 \mathrm{~S}^{-1}$ to zero. Indication of this is that the starch and the products derived thereof are characterized by non-Newtonian behavior. However, a close examination of the up and down flow curves revealed that these curves were not coincident in the case of native starch, the copolymer and the composite, indicating that these starch hybrids exhibit thixotropic behavior. On the other hand, with respect to the carbamoylethylated products derived from native starch, the copolymer and the composite were characterized by pseudoplastic behavior because the up and down flow curves were coincident. This concluded that introduction of carbamoylethyl along with carboxyethyl groups in the molecular structure of the products under investigation induces the ability of the macromolecules of these products to rebuild immediately after the applied force was removed.

\section{Apparent viscosity}

The apparent viscosities of the cooked solutions of native starch as well as those of poly (Aam)-starch graft copolymers, poly (Aam)-starch composites, carbamoylethyl poly (Aam)-starch graft copolymers and carbamoylethyl composites were measured at different rates of shear. The rate of shear ranges from 129 to $1290 \mathrm{~S}^{-1}$. The measuring temperature was $80^{\circ} \mathrm{C}$ and the concentration of starch products was $10 \%$.

Figure 4 shows the flow profile curves of the native starch, the copolymer and the composite before and after etherification. The latter, as already indicated involves carbamoylethylation and carboxyethylation.

Preparation of the copolymer and composite was carried out using Aam at a concentration of $10 \%$ ows. A perusal at these curves reveals that the apparent viscosity decreases by increasing the rate of shear regardless of the nature of the starch products used. Nevertheless, the apparent viscosity relies on the nature of starch. For a given rate of shear of up to $1000 \mathrm{~S}^{-1}$ the apparent viscosity follows the order: native starch >composite> copolymer> carbamoylethyl starch>carbamboylethyl composite> carbamoylethyl copolymer. This is also valid at rates of shear higher than $1000 \mathrm{~S}^{-1}$ except that the apparent viscosity of the carbamoylethyl composite becomes equal to the apparent viscosities of carbamoylethyl copolymer. 


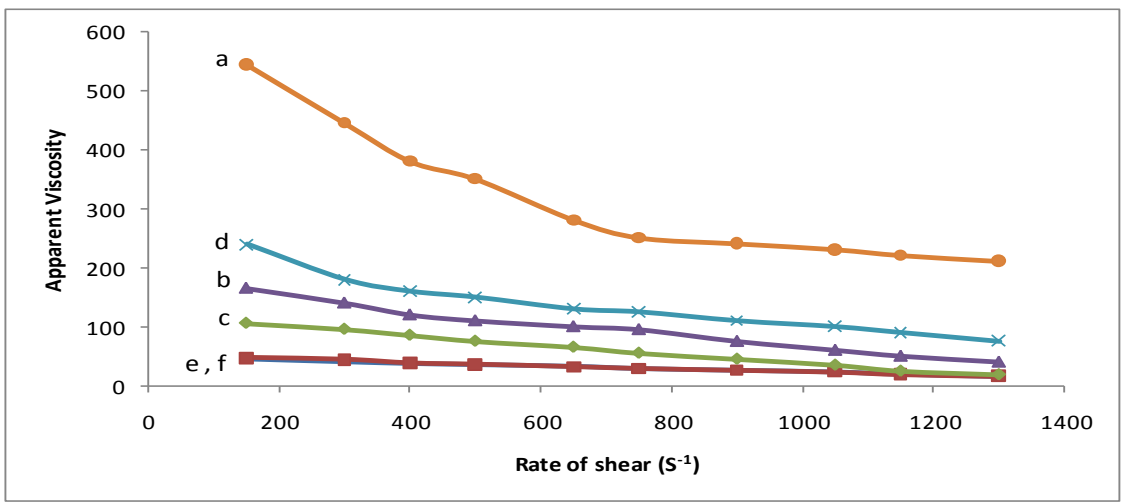

Fig. 4. Effect of rate of shear on the apparent viscosity of starch- poly (Aam) composite and copolymer before and after etherification with Aam in alkaline medium when $10 \%$ Aam was used in the polymerization reaction. a-Native Starch b- Carbomoylethyl starch c- Poly (Aam)- starch graft copolymer d- Poly(Aam)- starch composite e-Carbomoylethyl poly(Aam)- starch composite f- Carbomoylethyl-poly (Aam)- starch graft copolymer

The above order implies that the native starch undergoes oxidative degradation during the polymerization process under the influence of the initiator thereby decreasing the molecular size of starch. Such a decrease in combination with the increased solubility by the presence of poly (Aam) would account for the lower apparent viscosities of the composite and the graft copolymer when compared with native starch. Etherification causes further reduction in the molecular sizes of starch, composite and starch copolymer by oxidative degradation occurring during the carbamoylethylation and carboxyethylation process. Introduction of solubilizing groups such as carbamoylethyl and carboxyethyl groups will enhance the solubility. Hence the solubility and the low molecular mass of the etherified products are responsible for the decrease of the other apparent viscosities. It is known that the viscosity decreases when the solubility increases.

The decrease in apparent viscosity by increasing the rate of shear within the range studied (Fig. 4) deserves more classification. Shear thinning of polymer solution under investigation (containing long entangled and looping molecular chains) could be explained by considering the polymer solution before and after the shear is applied.

Before applying the shear, the polymer will maintain internal order and, correspondingly, a sizeable internal resistance against flow, i.e., higher viscosity. On the other hand, with increasing shear rates, rod like particles suspended in the liquid will be turned lengthwise into the direction of the flow. Chain type molecules in a solution can disentangle, stretch and orient parallel to the driving force. Particles or molecular alignment allow particles and molecules to slip past each other more easily. This shows up as reduced viscosity.

Egypt. J. Chem. 56, No. 4 (2013) 
The above shear thinning phenomenon in the field of rheology is called pseudoplasticity. This phenomenon is due to the gradual progressing structural breakdown upon deformation of the non-Newtonian system.

The decrease of pseudoplasticity by chemical modification of starch through graft copolymerization with Aam and etherification through reaction with acrylamide in alkaline medium, i.e. carbamoylethylation and carboxyethylation indicates that starch undergoes molecular changes under the influence of both grafting and etherification as detailed above. It is certain, however, that the magnitude of changes brought about by the etherification reaction is greater than the graft copolymerization reaction as evidenced by the lower value of the pseudoplasticity found with the former.

The above order signifies further that the presence of poly (Aam) chains with starch in the form of graft (chemically bonded) and /or in the form of homopolymer (physical entangled) lowers the mobility of the macromolecules. Thus the physical entanglement of the poly (Aam) chains with themselves as well as with starch hydroxyls together with long grafted chains and increased branching would explain the higher viscosities exhibited by the composite and the graft copolymer. On the other hand, introduction of carbamoyethyl and carboxyethyl groups in the starch backbone ruptures the physical forces (hydrogen bonds, van der Waal's forces) between the starch hydroxyls and starch-poly (Aam) chains. As a result, the viscosity is reduced.

\section{Summary and Conclusion}

Novel starch hybrids were synthesized through carbamoylethylation etherfication of poly (Aam)-starch graft copolymer and poly (Aam)-starch composite using Aam as the etherficaying agent in presence of $\mathrm{NaOH}$. During carbamoyethylation of the composite and the copolymer carboxyethylation inevitably occurs via conversion of the carbamoylethyl groups to carboxyethyl groups. Acrylamide moieties in the starch copolymer and the composite would similarly undergo alkaline hydrolysis to carboxyethyl groups. Dependence of the etherification (carbomoylethylation and carboxyethylation) on conditions used as well as of etherfication and on nature of starch substrate and the onset of this on the rheological properties of the newly synthesized starch hybrids bring into focus the following conclusions:

(1) The composite is less susceptible to carbomylethylation than the copolymer, suggesting that the homopolymer which is intimately associated with other components of the composite acts as a diffusion barrier for the etherifying agents to reach the reactive sites (hydroxyl groups) on the starch molecules.

(2) The magnitude of poly (Aam) content in the form of graft or homopolymer or both adversely affect the etherfication reaction indicating that the starch hydroxyls are the only groups amenable for etherfication. 
(3) In accordance with the above, the native starch is much more susceptible to carbomoylethylation than the copolymer and composite.

(4) Having studied the factors affecting etherfication of the copolymer, the following conditions were formulated to constitute optimal: $[\mathrm{NaOH}]=5 \%$ ows, $[$ Aam $]=100 \%$, Reaction time $=90 \mathrm{~min}$ and temperature $=30{ }^{\circ} \mathrm{C}$.

(5) Before etherfication the composite, the copolymer and the native starch exhibit non-Newtonian thixotropic behavior.

(6) After etherification, the etherified composite, the etherified copolymer and the etherified starch are characterized by non-newtonian pseudoplastic behavior, presence of the carbomoylethyl groups and the carboxyethyl groups seem to enhance the ability of the macromolecules of the starch hybrids in question to rebuild themselves immediately after deformation under the effect of the applied force.

(7) The apparent viscosity of the native starch, starch composite and starch copolymer decreases significantly after etherification for two reasons: first, the enhancement of solubility by virtue of the solubilizing action of the introduced groups (carbamoylethyl and carboxyethyl) in the molecular structure of the starch hybrids. Second, the fall in the molecular mass of starch under the oxidative action of alkali in presence of oxygen during the etherification reaction.

Acknowledgment: The authors are indebted to late Profs. Drs. A. Bayzeed and F. El Sisi of the Textile Research Division, National Research centre, for their sound contribution in this research.

\section{References}

1. Wurzburg, O.B., Introduction in Modified Starches: Properties and Uses, CRC Press, Florida, Chap.I. (1987).

2. Smith, J., Characterization and analysis of starches, p. 569 in Whistler and Paschall (Ed.) Starch: Chemistry and Technology, Academic Press, N.Y.vol.II (1967).

3. El-Sheikh, M.A., Ramadan, M.A. and El-Shafie, A., Photo oxidation of rice starch 1using hydrogen peroxide as a photo initiator. Carbohydrate Polymers, 80, 266-269 (2010).

4. El-Sheikh, M.A., Ramadan, M.A. and El-Shafie, A., Photo oxidation of rice starch. II. Using water soluble photo initiator. Carbohydrate Polymers, 78, 235-239 (2009).

5. Laignel, B., Bliard, C., Massiot, G. and Nuzillard, J.M., Proton NMR spectroscopy assignment of d-glucose residues in highly acetylated starch. Carbohydrate Research, 298, 251-260 (1997).

Egypt. J. Chem. 56, No. 4 (2013) 
6. Hebeish, A., Khalil, M.I. and Hashem, A., Carboxymethylation of starch and oxidized starches. Starch/Starke, 42, 185-190 (1990).

7. Hebeish, A., El Naggar, M.E., Fouda, M.G., Ramadan, M.A., Al-Deyab, S. S. and ElRafie, M.H., Highly effective antibacterial textiles containing green synthesized silver nanoparticles. Carbohydrate Polymers, 86, 936-940 (2011).

8. Hebeish, A., Higazy, A., El-Shafei, A. and Sharaf, S., Synthesis of carboxymethyl cellulose (CMC) and starch-based hybrids and their applications in flocculation and sizing. Carbohydrate Polymers, 79, 60-69 (2010).

9. El-Rafie, M.H., El Naggar, M.E., Fouda, M.G., Ramadan, M.A., Al-Deyab, S.S. and Hebeish, A., Environmental synthesis of silver nanoparticles using hydroxypropyl starch and their characterization. Carbohydrate Polymers, 86, 630-635 (2011).

10. Fanta, G.F. and Doane, W.M., Grafted Starches, In Modified Starches: Properties and Uses, Wurzburg, O.B. (Ed.), CRC Press: Boca Raton ,Florida (1986).

11. Meshram, M.W., Patil, V.V., Mhaske, S.T. Thorat, B.N., Graft copolymers of starch and its application in textiles. Carbohydrate Polymers, 75, 71-78 (2009)

12. Quan, Y., Kweaon, M.R. and Souslki, A., Effect of cross linking on functional properties of cationic corn starch. Starch/Starke, 49, 458-464 (1997).

13. Hebeish, A., Beliakova, M.K. and Bayazeed, A., Improved synthesis of poly (MAA)-starch graft copolymers. J.Appl.Polym.Sci. 68, 1709-1715(1998).

14. Khalil, I., Bayazeed, A., Farag, S. and Hebeish, A., Chemical modification of starch via reaction with acrylamide. Starch/Starke. 39, 311-315 (1987).

15. Pacsu, R. and Hiller, L.A., Cellulose studies: VI, determination of carboxyl groups in cellulosic materials. Text. Res. J. 16, 390-392 (1946).

16. Cole, J.O. and Parks, C.R., Semimicro-kjeldahl procedure for control laboratories.Ind. Eng. Chem. Anal. Ed. 18, 61 (I946). 


\section{متزاوجات جديدة من النشا بتكوين إثيرات من بوليمرات الأكريل أميد مع النشا}

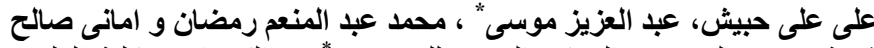
شعبة بحوث النسيج - المركز القومى للبحوث و وقتم الكيمياء - كلية العلوم -

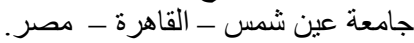

تم تخليق متز اوجات جديده من النشا تحتوى على مجمو عات الأكريلاميد على هيئة

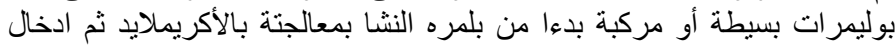

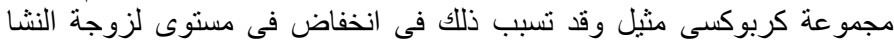

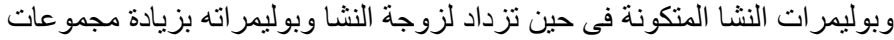
الكاربامويل مثيل والكاربوكسى اثيل فى جزيئات مثز اوجات النيات النشا . 